\title{
Aprendizaje Basado en Proyectos (ABP) y el método Jigsaw en el estudio del paisaje Project Based Learning (PBL) and Jigsaw method in landscape study
}

\author{
Departamento de Geografía \\ Universitat Rovira i Virgili \\ Vila-seca, España
}

Amalia Palacio Buendía, Yolanda Pérez Albert, David Serrano Giné

amalia.palacio@yahoo.es,myolanda.perez@urv.cat, dserrano@terrassa.uned.es

\begin{abstract}
Resumen- La presente comunicación es una propuesta metodológica para el estudio del paisaje en la enseñanza secundaria, a través de la combinación del Aprendizaje Basado en Proyectos (ABP) y el método Jigsaw. El ABP estimula la curiosidad del alumno, la creatividad y especialmente que se trabaje de forma activa en su aprendizaje. Se construye así, una guía estructurada conformada por diversas dinámicas cooperativas donde se utilizan técnicas de lectura compartida, el método Jigsaw y la elaboración de un mapa colaborativo. Como consecuencia de estas estrategias el estudiante trabaja en equipo, se organiza, clasifica y utiliza las Tecnologías para el Aprendizaje y Comunicación (TAC) e incluye en el proceso de aprendizaje la descripción del paisaje y técnicas de análisis espacial.
\end{abstract}

Palabras clave: Aprendizaje Basado en Proyectos (ABP), Aprendizaje cooperativo, Paisaje, Lectura Compartida, Método Jigsaw.

Abstract- This communication is a proposal about landscape study through the combination Project Based Learning (PBL) and Jigsaw method. The PBL promotes the student's curiosity, creativity and especially that they work actively on their learning. Thus, a structured guide made up of various cooperative dynamics is constructed where shared Reading Techniques, the Jigsaw Method and the elaboration of a collaborative map are used. As a consequence of these strategies, the student works as a team, organizes, classifies and uses Technologies for Learning and Communication (TAC) and includes landscape description and spatial analysis techniques in the learning process.

Keywords: Project Based Learning (PBL), Cooperative Learning, Landscape, Shared Reading, Jigsaw Method.

\section{INTRODUCCIÓN}

El aprendizaje y el desarrollo de habilidades sociales de estudiantes que aprenden en situaciones cooperativas son mejores que cuando este proceso se realiza de forma individual y competitiva (Mendoza Becerra, Cobos Lozada, \& Gómez, 2005). El aprendizaje cooperativo es una metodología docente innovadora que en el desarrollo de su aplicación el alumno forma parte activa del proceso de aprendizaje. También proporciona la adquisición de competencias básicas y transversales, habilidades para trabajar en equipo, destrezas de argumentación y para generar soluciones de puesta en común (Galindo \& De La Varga, 2016). Ramírez (2017) cita que el $\mathrm{ABP}$ es un marco alternativo a la enseñanza tradicional que se compromete con las necesidades formativas reales del aprendiz, lo entrena para habilidades de pensamiento elevadas y se trabaja desde el contexto cooperativo y real.

Del mismo modo, el método Jigsaw requiere en el proceso de aplicación que todos los miembros del equipo lleven a cabo sus responsabilidades particulares para así completar la tarea asignada (Gibbons, 2015). Anguas (2007) cita que con relación a esta técnica que "es una forma de evitar la conflictividad social en el aula". En esta misma línea, Novejarque Civera y Pisá Bó, (2017) estudian la confianza de la aplicación de la técnica Jigsaw y los Mapas Conceptuales para mejorar los resultados académicos del alumnado. Pozzi (2010) reflexiona sobre la estructura social del Jigsaw y determina que la forma de interacción interna y externa que se tiene con otros grupos, la estructura definida, los objetivos, la programación, los resultados y la cohesión de equipo es ideal para trabajar el aprendizaje colaborativo digital. El mismo autor indica que el método requiere una coordinación especial en cuanto a la organización y distribución de los equipos. Los grupos de expertos diseñados en la fase inicial deberían ser homogéneos en competencias, mientras que en la fase siguiente en el grupo base o grupo Jigsaw deberían ser heterogéneos. Es positivo que los participantes jueguen diferentes roles en ambos grupos. En el grupo de expertos todos tienen un mismo nivel de responsabilidad y en el grupo base cada persona es responsable de un segmento, la responsabilidad individual es alta y al final entienden que su contribución es indispensable, única y esencial para el éxito de la tarea.

La puesta en práctica del ABP y el método Jigsaw es una combinación novedosa que se puede complementar efectivamente en el aula y es por esta razón que se han elegido para la presente propuesta y no otras estrategias de carácter más sencillo y espontáneo.

El grupo seleccionado para la aplicación de la siguiente propuesta metodológica es $1^{\circ} \mathrm{ESO}$ y una ratio estimada de 25 alumnos. Los contenidos y estándares de aprendizaje inicialmente tienen como punto de partida los contenidos 
claves reflejados en el currículo (Decreto 187/2015) específicos de la competencia número 5 "explicar las interrelaciones entre los elementos del espacio geográfico, para gestionar las actividades humanas en el territorio con criterios de sostenibilidad".

Siguiendo esta línea de ideas se incluye en las competencias número 5 y 6 de las dimensiones geográficas del Decreto 187/2015 del currículum de educación secundaria obligatoria del ámbito de las ciencias sociales.

Los criterios de evaluación para $1^{\circ}$ ESO relacionadas con las propuestas son: "comparar y analizar los principales paisajes, distinguirlos en función de los elementos naturales y humanizados que interaccionan y caracterizar las formas de vida valoradas en su diversidad, así como los obstáculos y las oportunidades que posibilitan de acuerdo con los recursos que proporcionan" y así también, "diferenciar los recursos renovables y los no renovables, y los riesgos naturales y antrópicos. Identificar el impacto de la actividad humana sobre el territorio" (Decreto 187/2015).

Los conceptos que se deben delimitar y reconocer antes de comenzar la descripción de la propuesta son: El Paisaje, El Aprendizaje Basado en Proyectos y el Método Jigsaw.

\section{A. Paisaje}

El Consejo de Europa (2000) define el paisaje como un elemento clave para el bienestar social e individual de la población. Su protección, gestión y planificación implica derechos y responsabilidades por parte de todos. El paisaje se define como cualquier parte del territorio tal y como lo percibe la población, es el resultado de la acción de factores humanos y naturales y de las relaciones que se establecen entre ellos.

La identificación y caracterización del paisaje forma parte de la primera fase del esquema metodológico de todo catálogo del paisaje o de los planes territoriales de paisaje. En esta fase se identifican áreas del territorio con características semejantes. Para ello se consideran los elementos naturales, culturales y visuales que configuran el paisaje, así como el componente simbólico y perceptivo del mismo (Nogué i Font et al., 2014; Sabaté Bel and Vera Galván, 2008).

El estudio del paisaje contribuye positivamente a la formación ambiental del alumno tal como lo sugiere Casa Jericó, Puig i Baguer, \& Erneta Altarriba (2017).

\section{B. Aprendizaje Basado en Proyectos (ABP)}

El principal exponente del ABP es el psicólogo y filósofo John Deway, su doctrina se basa en el aprendizaje activo y en que se debe aprender haciendo, en un contexto de formación, experiencia, e interpretación de proyectos (Landron, Agreda Montoro, \& Colmenero Ruiz, 2018; Bosica, Pyper, \& MacGregor, 2021). Según Pereira Baz (2015) en el aprendizaje ABP, el papel del profesor cambia y pasa de ser un simple transmisor de información a ser un facilitador $y$ orientador, de modo que los alumnos asumen el peso del aprendizaje y se trabaja a partir de la cooperación, siempre manteniendo la vinculación con el currículo. El alumno no memoriza, comprende el problema planteado y aprende con mayor intensidad, ya que él es el que hace y construye (Edutopia, 2013).

La orden ECD/65/2015, 2015 a nivel metodológico señala que las técnicas de ABP "favorecen la participación activa, la experimentación y un aprendizaje funcional”. Según Benejam et al. (1997) el ABP se caracteriza porque se plantea a los alumnos un tema a desarrollar o que también ellos podrían seleccionar o escoger en función de sus intereses, a partir de aquí, se planifica y organizan un conjunto de actividades para alcanzar los objetivos del proyecto, se lleva a cabo la idea y se presenta a la clase (o a otras instancias como al colegio, familiares e incluso a otros grupos de estudiante). Los alumnos trabajan con autonomía y en grupos reducidos, el profesor les orienta y les suministra los recursos.

\section{Método Jigsaw}

El método Jigsaw de Eliot Aronso sigue una estructura de tarea cooperativa, es fácil de aplicar y adaptar (Santos-Rego, Lorenzo-Moledo, \& Maño, 2009). Se define como una estructura de aprendizaje cooperativo compleja, que divide el material o los recursos a aprender en partes moderadas entre los integrantes de un equipo base (Galindo \& De La Varga, 2016). Acto seguido, se constituyen grupos de expertos para reflexionar y profundizar en cada tema u objeto de estudio. Por último, estos grupos de expertos regresan al grupo original o base para que cada integrante explique los conocimientos adquiridos.

El grupo base preferiblemente es reducido. El número de integrantes tiene que coincidir con el número de partes en las que se divide el material. Igualmente, es significativo que cada equipo establezca roles o funciones a cada integrante, en tal caso, podría haber en cada equipo como sugerencia un coordinador, un secretario, un corrector y un portavoz-coach. Cada una de estas funciones se tienen que explicar y se pueden adaptar según las circunstancias reales del aula.

Tanto el método Jigsaw como otras estrategias de aprendizaje cooperativo se basan en cinco pilares fundamentales que son la interdependencia positiva, la responsabilidad individual y grupal, la interacción promotora, habilidades sociales y análisis de grupo (Galindo \& De La Varga, 2016). El procedimiento a seguir para llevar a cabo la mencionada técnica es el siguiente; organización de los equipos bases; reparto del material; estudio individual; reunión de expertos; preparación individual; explicaciones del grupo base; integración y solución de la actividad; y finalmente la evaluación (Valero García \& Vaquerizo García, 2009; Wilson, Pegram, Battise, \& Robinson, 2017)

\section{CONTEXTO}

El objetivo principal de este trabajo es exponer y comprender los criterios de actuación necesarios para la elaboración de un proyecto en el marco de la temática del paisaje basado en la aplicación de metodologías activas. Para ello se propone 
utilizar el Aprendizaje Basadas en Proyectos (ABP), en combinación con otras metodologías activas como la Lectura Compartida, el Método Jigsaw y la implementación de la cartografía colaborativa. Se dirige a estudiantes de $1^{\circ}$ ESO de la asignatura de Geografía e Historia. Aunque, estudiar los tipos de paisajes $\mathrm{y}$ cómo estos son transformados constantemente por la sociedad son temáticas de estudio que también se abordarán y estudiarán en $3^{\circ} \mathrm{ESO}$.

La elección y justificación ha resultado del Decret 143/2007, 2007. En el presente Decreto se establece la ordenación de las Enseñanzas de Educación Secundaria y Obligatoria y en el apartado de ciencias sociales, geografía e historia en la sección de las contribuciones o aportaciones de la geografía a las ciencias sociales, cita que la educación geográfica permite que las personas se apropien de los espacios y medios donde desarrollan su vida diaria, así también a aprender apreciar los paisajes. Además, en los contenidos comunes de toda la etapa se incluyen localizar y caracterizar los diferentes tipos de paisaje y analizar la interacción entre las sociedades y el medio.

Actualmente, los jóvenes se comunican en diversas ocasiones a través de redes sociales y fotografías. Especialmente capturan imágenes de lugares visitados y de experiencias vividas únicas y especiales que quieren compartir. Un gran número de ellas corresponden al disfrute de un paisaje en concreto.

El presente trabajo contribuye en la mejora del aprendizaje del paisaje porque se realiza a partir de estrategias cooperativas y de la vivencia del alumno. Estudios del paisaje en la educación secundaria plantean la necesidad que hay de educar y sensibilizar a la ciudadanía en materia de paisaje, así también se ha analizado en diversas investigaciones el tratamiento que se le da al paisaje en los libros de textos escolares (Barbero, 2016; Casas Jericó \& Erneta Altarriba, 2015). Por consiguiente, los discentes cuando observen y aprecien un paisaje en una fotografía no solo pensaran en la localización, lugar donde se realizó o persona que aparece, sino también, tendrán inquietudes de razonar, meditar u opinar sobre la conservación del paisaje de dicho territorio, en recuperar las áreas degradadas, o en reducir el impacto que provoca determinada actividad antrópica.

\section{DESCRIPCIÓN}

El presente ABP se estructurará en tres fases (Contreras Jordan \& Gutierrez Díaz del Campo, 2017) y cinco sesiones de 50 minutos cada una, en las que se realizarán las siguientes actividades:

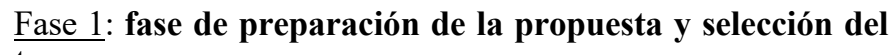 tema.}

La primera sesión es introductoria y en ella se realizará una lectura compartida y una explicación general del proyecto por parte del profesor. Las estructuras simples cooperativas como la lectura compartida aseguran una mejor interacción del equipo de trabajo (Montoro Cabrera, 2009).
La lectura compartida se podría realizar en grupos de cuatro personas. Para llevarlo a cabo un alumno comienza la lectura del primer párrafo, el estudiante de su derecha explica con sus propias palabras la lectura previa, y prosigue a leer el segundo párrafo y a continuación, el compañero de su derecha explica el párrafo número dos y así sucesivamente has terminar la lectura.

La introducción del tema por parte del profesor se realiza empleando la exposición oral de los conceptos claves, y con el uso de diapositivas explicativas, diagramas y fotografías. Esta sesión se aprovecha para explicar los métodos cooperativos a utilizar para trabajar los contenidos de paisaje, la organización de los grupos y se explican las siguientes cuatro sesiones con un esquema guía.

Al finalizar la sesión los alumnos tendrán una idea estructurada y amplia de la temática a trabajar en las siguientes sesiones.

\section{Fase 2: fase de desarrollo}

En la segunda sesión se crean los grupos bases (o grupos Jigsaw) de cuatro integrantes siguiendo un criterio de heterogeneidad entre los alumnos, en cuanto a género, habilidades, y otros juicios que se consideren oportunos. Luego se reparte el material segmentado, o subtema que estudiará cada integrante.

A modo de ejemplo, los subtemas son: concepto del paisaje; clasificación del paisaje según las características del geosistema; clasificación del paisaje según su funcionalidad; tipos de paisaje en función de la intervención humana.

A continuación, se crean grupos de expertos por subtema, cada grupo debe elaborar un esquema explicativo de su temática, podrían llamarse fichas, las cuales se elaborarán con bubbl.us, esta aplicación facilita la elaboración de mapas conceptuales. Así también, se tiene que profundizar en el subtema a través de búsqueda en internet y discusión de equipo.

El aprendizaje cooperativo a partir del método sigue la dinámica de un puzzle tradicional. Ahora bien, en la sesión número tres se tienen que configurar nuevamente los grupos base o Jigsaw de manera que se regresa a los originales grupos de trabajo, considerando que hay un experto de cada tema.

El objetivo de cada grupo base es estudiar el paisaje a partir de la descripción de fotografías y utilizando los conocimientos previos adquiridos. De esta manera se podría estudiar el paisaje de un parque natural, de una ciudad, de un pueblo o de un determinado lugar de interés del alumnado, podría ser el paisaje de la residencia habitual del estudiante.

El resultado final será un poster elaborado con la herramienta Canva en el que aparezcan las fotografías previamente seleccionadas con la descripción y clasificación del paisaje que muestran.

En la cuarta sesión cada grupo de trabajo tiene que aportar dos fotografías representativas de un paisaje que simbolice el lugar de estudio. Es necesario que sean fotografías amplias; que abarquen una gran cantidad de elementos propios de ese paisaje. Estas fotografías las pueden obtener de Internet 
(siempre respetando la propiedad intelectual de las mismas, se tiene que indicar la fuente y el autor de estas) o las pueden realizar los mismos estudiantes con una cámara fotográfica o móvil. El profesor actuará de guía y apoyo en esta actividad.

Información relevante de las fotografías:

- Dirección localización aproximada. La ubicación se puede realizar mediante la dirección postal o coordenadas $\mathrm{x}, \mathrm{y}$ dependiendo del tipo de ámbito urbano o natural o de los objetivos pedagógicos planteados

- Fecha de la imagen. Es importante tener detalles de la fotografía y que esta sea actual. Teniendo en cuenta esta característica, sería posible realizar análisis cronológicos comparados.

- Elementos del paisaje de la fotografía y clasificación (por ejemplo, tipos de paisaje según su funcionalidad).

- Alguna observación que considere relevante de los lugares donde se tomó la fotografía o que se citaba en el lugar de Interne donde se adquirió.

Estas fotografías se tienen que geolocalizar en el mapa (Buzo, 2013). En la construcción del mapa colaborativo de paisaje es aconsejable que se cuente con un número de ordenadores adecuados y conexión a Internet. Se puede emplear para ello el aula de informática de la institución.

La construcción del mapa colaborativo se realiza con MyMaps. Todos los grupos deben trabajar en el mismo espacio de trabajo (es decir que el mapa esté compartido entre los integrantes del equipo). Posteriormente, se abre una discusión sobre los paisajes identificados, las posibles intervenciones a las que ha sido sometido dicho espacio y cuales evidencian mayor impacto ambiental, turístico o social.

\section{Fase 3: fase de comunicación y evaluación}

La quinta sesión, al igual que todas las demás es evaluativa. Pero en esta se evalúan todos los materiales elaborados en su conjunto la presentación oral del esquema, la infografía y el mapa colaborativo. Se finaliza la evaluación con un examen tipo test. Se debe valorar también lo que se ha aprendido y reflexionar sobre posibles adaptaciones o mejoras de la metodología propuesta.

\section{RESUltados Y CONCLUSIONES}

Diversas investigaciones dan por sentado los amplios beneficios que tiene la aplicación del ABP y las técnicas cooperativas como el Jigsaw, aunque ninguna de las pesquisas las combinaba. Diversos autores mencionan ventajas en cuanto a la implicación, motivación, aumento cognitivo, mejoras en la interacción del alumnado y de los resultados académicos (Anguas et al., 2007; Contreras Jordan \& Gutierrez Díaz del Campo, 2017; Galindo \& De La Varga, 2016; Pozzi, 2010; Valero García \& Vaquerizo García, 2009; Wilson et al., 2017)
Los resultados esperados con la aplicación de esta metodología dan respuesta al objetivo de aprendizaje de comprender el concepto y los elementos del paisaje, a interiorizar y relacionar con facilidad el contenido gracias al carácter vivencial del proyecto y a mejorar la interacción y confianza del discípulo.

Se espera que el alumno integre la información del paisaje después de su aplicación con las de compañeros de otros grupos y así construir un amplio conocimiento con ejemplos prácticos.

Así también, esta metodología permite el desarrollo de tareas cortas que se evalúan por medio de la evaluación formativa en cada sesión, que la función del profesor sea de mediador y guía en el transcurso del proyecto, permite fomentar la autonomía del alumno y una mejor gestión del aula; además, refuerza la autoestima y motivación del estudiante.

El trabajo cooperativo con una buena estructura y organización es un incentivo innovador en el desarrollo del ABP. Se han consultado proyectos que utilizaban las ventajas de estas herramientas(Pozzi, 2010; Santos-Rego et al., 2009), pero la presente combinación metodológica resalta una aportación integral y ajustada a la realidad educativa de los contenidos curriculares. En estos proyectos de Jigsaw y ABP se combinaban solamente en la fase de trabajo con los mapas conceptuales (Valero García \& Vaquerizo García, 2009).

A nuestro conocer, esta es la primera aplicación del método Jigsaw que se realiza para estudiar el paisaje. Este método ha sido utilizado en un abanico de investigaciones del ámbito de la educación, es factible su aplicación en las ciencias sociales $\mathrm{y}$ es viable de adaptar y replicar en contextos similares al presentado en esta comunicación.

En este orden de ideas, desarrollar una buena práctica para identificar el paisaje a partir de la fotografía y la cartografía colaborativa es una experiencia enriquecedora que podría ser continuada en cursos posteriores y así también, se podría aumentar el nivel de complejidad.

Ampliamente se reconoce que la implicación de los alumnos en proyectos donde el espacio estudiado es conocido por ellos aumenta, y con ello también la disposición de ofrecer ideas, propuestas, alternativas y soluciones.

Para finalizar, se tiene que destacar la mayor exigencia que se requiere del profesorado para la elaboración y aplicación de estos proyectos, pero que sin duda tienen resultados ventajosos a largo plazo.

\section{AGRADECIMIENTOS}

Agradezco a la facultad de Turismo y Geografía de la Universitat Rovira i Virgili y al Grupo el GRATET por la sinergia y años de investigación compartida. Al departamento de Educación de la Universidad Isabel I porque disfruté gratamente al cursar el Máster de Formación del Profesorado. 


\section{REFERENCIAS}

Anguas, J., Díaz, L., Gallego, I., Lavado, C., Reyes, A., Rodríguez, E., ... Valero, M. (2007). La técnica del Puzzle al servicio del aprendizaje de la programación de ordenadores. XII Jornadas de Enseñanza Universitaria de Informática [XII Meeting of Informatics University Teaching], 1-8. Retrieved from http://epsc.upc.edu/projectes/usuaris/miguel.valero/mate riales/docencia/articulos/jenui2006.pdf

Barbero, G. N. (2016). Análisis de la práctica educativa con SIG en la enseñanza de la geografía de la educación secundaria. Un estudio de caso en Baden- Württemberg, Alemania.

Benejam, P., Pagès, J., Comes, P., \& Quinquer, D. (1997). Enseñar y aprender ciencias sociales, geografía $e$ historia en la educación secundaria. (Institut de Ciencies de l'Educació Universitat de Barcelona, Ed.). Barcelona: Horsori.

Bosica, J., Pyper, J. S., \& MacGregor, S. (2021). Incorporating problem-based learning in a secondary school mathematics preservice teacher education course. Teaching and Teacher Education, 102, 103335. https://doi.org/10.1016/j.tate.2021.103335

Buzo, I. (2013). Introducción a la cartografía colaborativa en la educación secundaria. In XI Congreso Nacional de Didáctica de la Geografía (pp. 129-140).

Casa Jericó, M., Puig i Baguer, J., \& Erneta Altarriba, L. (2017). El "paisaje" como recurso para la Educación Ambiental. Experiencia práctica en el Equipamiento "Sendaviva" (Navarra). Observatorio Medioambiental, 20(0), 111-136. https://doi.org/10.5209/obmd.57949

Casas Jericó, M., \& Erneta Altarriba, L. (2015). El paisaje en la Educación Secundaria Obligatoria. Una oportunidad educativa en el cambio curricular LOE-LOMCE. Didáctica Geográfica, 16, 45-71.

Contreras Jordan, O., \& Gutierrez Díaz del Campo, D. (2017). El aprendizaje basado en proyectos en educación física. Barcelona: INDE.

Council of Europe. (2000). European Landscape Convention. Report and Convention Florence, ETS No. 17(176), 8. https://doi.org/http://conventions.coe.int/Treaty/en/Treat ies/Html/176.htm

Decret 143/2007. (2007). Decret 143/2007 del 29 de junio pel qual s'estableix l'ordenació dels ensenyaments de l'educació secundaria obligatoria.

Decret 187/2015. (n.d.). Decret 187/2015 del 25 d'agost d'Ordenació de l'Ensenyament Secundari Obligatori. Diari Oficial de La Generalitat de Catalunya, 6945, 1305.
ECD/65/2015. (2015). Orden ECD/65/2015, de 21 de enero, por la que se describen las relaciones entre las competencias, los contenidos y los criterios de evaluación de la educación primaria, la educación secundaria obligatoria y el bachillerato. Boletín Oficial Del Estado, (25), 6986-7003. Retrieved from https://www.boe.es/boe/dias/2015/01/29/pdfs/BOE-A2015-738.pdf

Edutopia. (2013). Los diez consejos principales para evaluar el aprendizaje basado en proyectos, 1-14.

Galindo, F., \& De La Varga, J. (2016). El Método Puzzle Como Técnica Para El Aprendizaje Cooperativo De Los Mapas Estratégicos: Una Experiencia En La Asignatura " Administración De Organizaciones ". Docencia: Metodología Y Experiencias Docentes, 10.

Gibbons, P. (2015). Scaffolding language, scaffolding learning. Portsmouth.

Landron, M. L., Agreda Montoro, M., \& Colmenero Ruiz, M. J. (2018). El efecto del aprendizaje basado en proyectos en estudiantes con altas capacidades intelectuales de una segunda lengua. Revista de Educacion, 2018(380). https://doi.org/10.4438/1988-592X-RE-2017-380-378

Mendoza Becerra, M., Cobos Lozada, C., \& Gómez, L. (2005). Aprendizaje cooperativo soportado por computador basado en el método jigsaw. Revista UIS Ingenierias, 4(2), 85-98.

Montoro Cabrera, M. del C. (2009). El aprendizaje cooperativo: Un instrumento de transformación para la mejora de la calidad de la enseñanza. Caleidoscopio, Revista Digital de Contenidos Educativos, (2), 8. Retrieved from http://dialnet.unirioja.es/servlet/articulo? codigo $=317631$ $4 \&$ info $=$ resumen\&idioma $=\mathrm{ENG}$

Nogué i Font, J., Sala i Martí, P., Aragonès, J., Saladié, S., Sabaté, X., Grau Oliveras, J., ... Losantos, À. (2014). Catàleg de paisatge Les Terres de l'Ebre.

Novejarque Civera, J., \& Pisá Bó, M. (2017). Herramientas de innovación docente en grupos reducidos: aprendizaje cooperativo y mapas conceptuales. https://doi.org/10.26754/cinaic.2017.000001_149

Pereira Baz, M. A. (2015). Los 7 elementos esenciales del ABP. Retrieved from http://cedec.educalab.es/7elementos-esenciales-del-abp/

Pozzi, F. (2010). Using Jigsaw and Case Study for supporting online collaborative learning. Computers and Education, $55(1)$, $67-75$. https://doi.org/10.1016/j.compedu.2009.12.003

Sabaté Bel, J., \& Vera Galván, J. R. (2008). Aspectos varios de la implementación de la Convención Europea de Paisaje en el Plan Territorial Especial de Ordenación de Paisaje de Tenerife. Cuadernos Geográficos, 5462(43), 51-67. 
Santos-Rego, M. A., Lorenzo-Moledo, M. D. M., \& Maño, D. P. C. (2009). Aprendizaje cooperativo: Práctica pedagógica para el desarrollo escolar y cultural. Magis, 1(2), 289-303.

Valero García, M., \& Vaquerizo García, M. B. (2009). Puzzles mejorados con mapas conceptuales. Jornadas de Enseñanza Universitaria de La Informática (JENUI), 8$10 . \quad$ Retrieved from http://upcommons.upc.edu/handle/2099/7876

Vergara Ramírez, J. J. (2017). Aprendo porque quiero. El
Aprendizaje Basado en Proyectos (ABP), paso a paso. (Ediciones SM, Ed.). Madrid.

Wilson, J. A., Pegram, A. H., Battise, D. M., \& Robinson, A. M. (2017). Traditional lecture versus jigsaw learning method for teaching Medication Therapy Management (MTM) core elements. Currents in Pharmacy Teaching and Learning, 9(6), 1151-1159. https://doi.org/10.1016/j.cpt1.2017.07.028 